\title{
Acceleration effects of microbial inoculum on palm oil mill organic waste composting.
}

\begin{abstract}
ABSTRACT The acceleration effects of inoculum in composting of empty fruit bunches were investigated. Composting of empty fruit bunches fibres in two sizes, $4 \mathrm{~cm}$ and $2 \mathrm{~cm}$ length, were treated with microbial inoculum consisting of Agromonas, Aspergillus, Azotobacter, Bacillus, Celhdomonas, Chaetomium, Clostridium, Coprinus, Microbispora, Penicillium, Pseudomonas, Thermoactinomyces, Trichoderma and Trichurus in separate laboratory scale in-vessel of 30 liters volume. A control without inoculum with $4 \mathrm{~cm}$ length empty fruit bunches was also conducted in parallel. The compost piles were shift-turned weekly. Parameters such as moisture content, temperature, $\mathrm{pH}$, and electrical conductivity were used to monitor the composting processes. The carbon-nitrogen ratio, UV-vis spectrophotometer test, and germination test were used to assess the maturity of compost. The results showed that the inoculum was effective in reducing the $\mathrm{C} / \mathrm{N}$ ratio by $54 \%$ compared to control $46 \%$ and rapidly increasing the UV-vis absorption ratio in first three weeks. By using functional microbes, the composting of empty fruit bunches was reduced to 5 weeks compared to 9 weeks for those without inoculation. The acceleration effect was more prominent for the $2 \mathrm{~cm}$ length samples.
\end{abstract}

Keyword: Palm oil; Mill; Waste; Empty fruit bunch; Effluent; Microbial inoculum. 